\title{
Acellular Mineralized Exoskeleton Shrimp (MES): A Natural Source of Bioactive Factors for Tissue Regeneration-Preliminary Results
}

\author{
Raquel Zita Gomes ${ }^{1,2}$, Rodrigo Valdoleiros e Silva 3 ,4,5,6, Américo Afonso ${ }^{7}$, \\ Maria Helena Fernandes ${ }^{8}$, Mário Vasconcelos ${ }^{9}$, José Cavalheiro ${ }^{10}$ \\ ${ }^{1}$ Faculty of Dental Medicine, University of Porto, Porto, Portugal \\ ${ }^{2}$ Private Practice (Raquel Zita Dental Clinic), Porto, Portugal \\ ${ }^{3}$ i3S-Institute for Research and Innovation in Health, University of Porto, Porto, Portugal \\ ${ }^{4}$ INEB - National Institute of Biomedical Engineering, Porto, Portugal \\ ${ }^{5}$ ICBAS-Institute of Biomedical Sciences Abel Salazar, University of Porto, Porto, Portugal \\ ${ }^{6}$ School of Medicine, University of Minho, Braga, Portugal \\ ${ }^{7}$ Department of Histology, Faculty of Dental Medicine, University of Oporto, Porto, Portugal \\ ${ }^{8}$ Department of Pharmacology, Faculty of Dental Medicine, University of Oporto, Porto, Portugal \\ ${ }^{9}$ Department of Biomaterials, Faculty of Dental Medicine, University of Oporto, Porto, Portugal \\ ${ }^{10}$ Faculty of Engineering, University of Oporto, Porto, Portugal \\ Email: raquelzitagomes@hotmail.com
}

How to cite this paper: Gomes, R.Z., Valdoleiros e Silva, R., Afonso, A., Fernandes, M.H., Vasconcelos, M. and Cavalheiro, J. (2022) Acellular Mineralized Exoskeleton Shrimp (MES): A Natural Source of Bioactive Factors for Tissue Regeneration-Preliminary Results. Journal of Biosciences and Medicines, 10, $34-43$.

https://doi.org/10.4236/jbm.2022.103005

Received: January 19, 2022

Accepted: March 1, 2022

Published: March 4, 2022

Copyright $\odot 2022$ by author(s) and Scientific Research Publishing Inc. This work is licensed under the Creative Commons Attribution-NonCommercial International License (CC BY-NC 4.0). http://creativecommons.org/licenses/by-nc/4.0/ (c) (i) (8) Open Access

\begin{abstract}
Current challenges in the development of scaffolds for bone regeneration include the engineering of biomaterials that can withstand a natural dynamic physiology on the bone that provides a matrix capable of supporting cell migration and tissue ingrowth. The objective of the present work was to develop and characterize a new biomembrane-Mineralized Exoskeleton Shrimp (MES) developed from the exoskeleton of paleomonetes. The integration of MES as a biomaterial for tissue regeneration relies on the growing evidence that the shrimp is characterized by a hierarchically arranged chitin fiber structure, mineralized predominately by calcium carbonate and/or calcium phosphate, bringing beneficial effects in bone regeneration. Additionally, the tridimensional MES structure, can act as a "tent" for Guided Bone Regeneration (GBR). Recently, our team has characterized the MES biomaterial by in vitro (human osteoblastic cellular cultures and immersion of the membrane in modified synthetic plasma) and in vivo (soft tissue in lab mice and hard tissue in rabbit model). The cellular growth in the MES membrane was very exuberant in cellular culture with osteoblastic colonization on its surface (histophilic and biocompatible). After the immersion in modified synthetic plasma for one week, a mass mineralization occurred throughout the membrane's surface (bioactive). The analysis of histological samples from experimental
\end{abstract}


surgery in lab mice showed that the MES membrane wasn't toxic to soft tissues and that it caused a moderate inflammatory response (first reabsorption signs at 8 weeks). The MES could act as a cell-guiding template that contains the necessary cues and adequate three-dimensional set to facilitate cell adhesion and promote tissue regeneration upon implantation and subsequent biodegradation.

\section{Keywords}

Guided Bone Regeneration, Biomaterial, Biocompatibility, Oral Surgery

\section{Introduction}

A big challenge in the development of scaffolds for oral and maxillofacial surgery, includes the engineering of biomaterials that promote an effective tissue guided regeneration. Recent research of "guided bone regeneration" hypotheses forced the need of new paradigms about the nature of the technique. We aim to propose to include biomembranes, which have the objective of functioning as a "tent" and as an orientation to the Guided Bone Regeneration (GBR) [1].

Guided Bone regeneration is a "surgical procedure that aims to separate the tissues through a natural barrier, periosteum, or artificial, in order to obtain the desired regeneration site, and not repair" [2]. The exclusion of unwanted tissue in the area where you want to form a specific tissue is the basic principle of guided regeneration. The aim is to allow only cells with bone regenerative capacity to have access to the region [3]. This objective can be achieved by local isolation with a physical barrier excluding unwanted tissues [3] [4] [5].

Nowadays, are currently available on the market various types of biological membranes for clinical use that constitute two major groups: the resorbable membranes and the non-resorbable. In the first group we have as main examples collagen membranes, polylactic acid, polyglycolic acid, poliglatin 910 and autologous fibrin. In the group of non-resorbable we can include the polytetrafluoroethylene (PTFE), expanded polytetrafluoroethylene, (e-PTFE) and titanium-mesh. The membranes for guided regeneration must have acceptable handling properties, being able to support the tissue, preserve and maintain the space, conformability to the shape of the defect, and have the characteristic that can be tailored to unique situations. The membranes should be easy to cut and shape and should not have sharp edges to avoid perforation of the tissue and allow an exact adaptation to a variety of morphological defects [6]. In addition to these general characteristics, resorbable membranes should be nontoxic, non-antigenic and produce minimal inflammatory response to the bioresorption process [7]. Several comparative tests between resorbable and non-resorbable membranes, both clinical and experimental, have been made to determine what type of membrane produces the greatest amount of bone tissue and the lowest postoperative degree of prob- 
lems [8] [9] [10] [11]. The results have not been entirely conclusive, not allowing finding what is the best type of membrane to be used in clinical practice, resulting in a virtual dead heat between these two types of membranes [6] [12].

The shrimps' exoskeletons are basically consisting of an organic part (chitin) and an inorganic matrix predominately formed by calcium carbonate and/or calcium phosphate, with an elastic behavior which can be beneficial for membrane tissue mechanical adaptation. The use of this mineralized membrane will function as a protective layer but also as a bone support [13]. In a previous study it was determined the overall strength of the membrane having a thickness of $0.25 \mathrm{~mm}$, obtaining an average value of $22 \mathrm{~N} / \mathrm{mm}^{2}$ [14]. In this research work, the goal was to study the biological characteristics of a new biomembrane developed from the exoskeleton of paleomonetes (Figure 1).

The objectives of this study were defined to enlighten:

- The biocompatibility of the membrane in osteoblastic cellular cultures.

- The bioactivity (mineralization capacity) of the membrane when immersed in synthetic modified plasma.

- The membrane behavior when in contact with soft tissues (muscular and subcutaneous) in the mouse.

- The existence of total or partial resorption and the quantification of that same resorption.

- The membrane biocompatibility when in contact with a bone defect and the real bone regenerative capacity, in the rabbit.

- The comparison of results both in vitro and in vivo between the test membrane and the control membrane.

To enlighten the topics already mentioned in the objectives of this study, the following experimental protocols were carried out.

\section{Materials and Methods}

\subsection{MES Preparation}

To remove the proteins, present in the exoskeletons of shrimp abdomens, we proceeded with a treatment of deproteinization, which consists in immersing the samples in a basic solution of sodium hydroxide at $3 \%(\mathrm{w} / \mathrm{w})$ heated to $70^{\circ} \mathrm{C}$

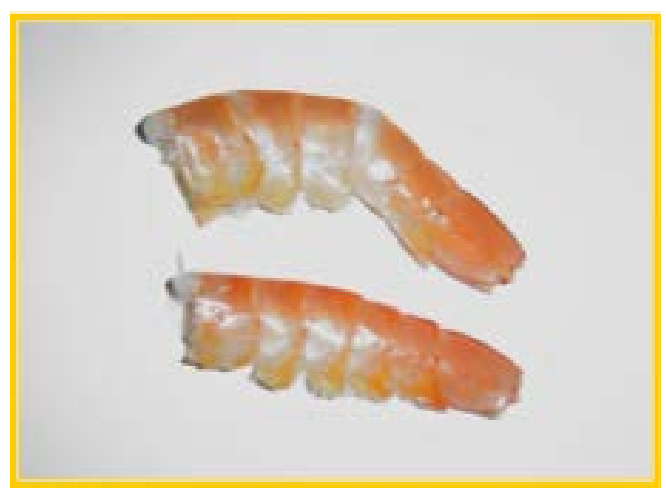

Figure 1. Exoskeletons of shrimp abdomens prior to treatment. 
with constant stirring. Samples were treated with about 10 solution changes until a whitish present salmon coloration. Then the shrimp skins were washed several times with deionized water, to be cleared of any tissue debris from the sodium hydroxide. Finally, the samples were passed through a sonicated apparatus for 15 minutes. Subsequently the membranes were subjected to a surface modification treatment, which allowed the introduction of silicon in their composition. This impregnation of silicates allows the surface to become bioactive (Figure 2).

\subsection{MES In Vitro Characterization}

Human osteoblastic cellular cultures to study the biocompatibility of the membrane in bone tissue in vitro. With cellular cultures, the aim was to verify and quantify the osteoblastic growth in the presence of the membranes. As control material in cellular cultures two absorbable collagen membranes already available in the market (Bio-guide ${ }^{\circledR}$ with parallel collagen fibers and $\mathrm{RCM}^{\circledR}$ of $\mathrm{ACE}^{\circledR}$ with collagen fibers cross-linked) were used.

Immersion of the MES test membrane in modified synthetic plasma in vitro to verify the mineralization degree of the membrane (bioactivity). Experimental surgery with 4 Mus Musculus mice, with implantation of the MES and collagen membranes in muscular and subcutaneous tissues with assessment of tissue reaction. At the end of 8 and 16 weeks the mice were euthanized (Figure 3, Figure 4).

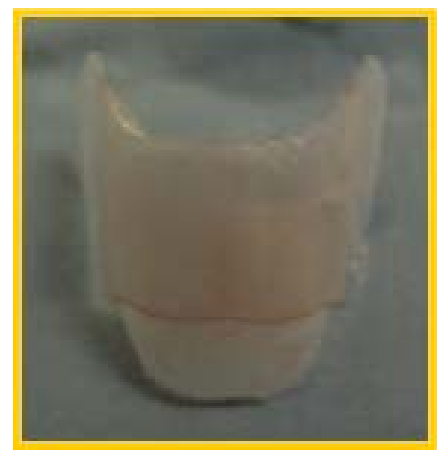

Figure 2. Macroscopic aspect of MES membrane after chemical treatment.

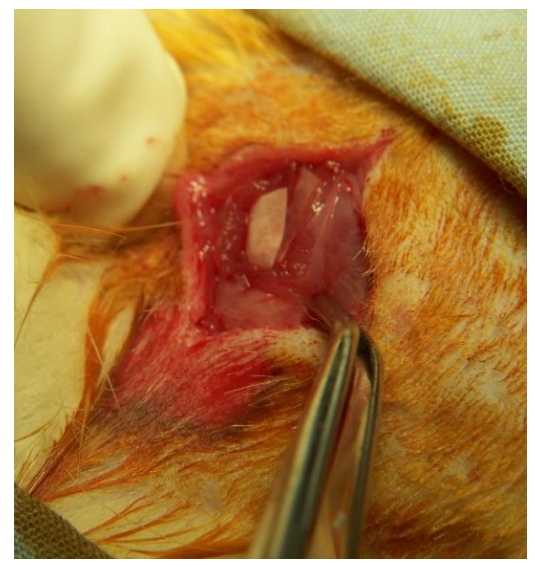

Figure 3. Experimental surgery on lab mice. 


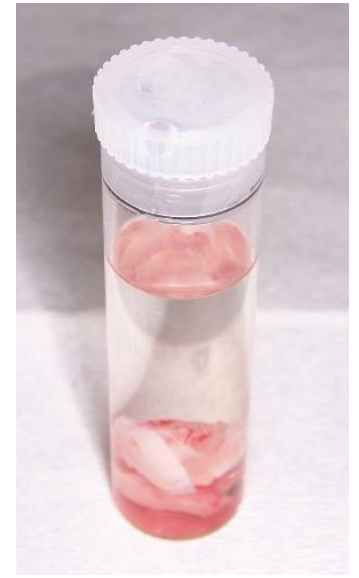

Figure 4. Histological sample of the mice after euthanasia.

\subsection{MES In Vivo Characterization}

Experimental surgery with 8 Huila rabbits to compare the bone neoformation with and without the MES membrane after the implantation in the surface of a critical size bone defect created in tibial cortical surface of the rabbit. The rabbits were euthanized after 4 and 8 weeks. In experimental surgery to test the regenerative bone capacity in the Huila rabbit, the control was made by a bone defect (control window) without any regenerative material, to conclude about the true regenerative capability of the MES membrane by comparison (Figure 5, Figure 6). The samples resulting from studies in vitro and in vivo were prepared histologically and visualized with optical microscopy, confocal microscopy, electronic scan microscopy and X-ray microanalyses.

\section{Results}

The cellular growth in the MES membrane was very exuberant from 21 days of cellular culture and clearer at 35 days (Figure 7, Figure 8). The MES membrane allowed growth and osteoblastic colonization on its surface (histophilic and biocompatible). After the immersion in modified synthetic plasma during one week of the MES membrane, a mass mineralization occurred throughout the membrane's surface (bioactive) (Figure 9). The analysis of histological samples from experimental surgery in lab mice showed that the MES membrane wasn't toxic to soft tissues and that it caused a moderate inflammatory response (first reabsorption signs at 8 weeks) (Figure 10, Figure 11). The analysis of histological samples from experimental surgery in lab rabbits showed that the MES membrane induced GBR in the created critical size bone defect in a faster and more effective way than the physiological regeneration occurred in the control bone defect (first signs of reabsorption at 8 weeks).

\section{Discussion}

In comparison with the other two collagen membranes, the cellular growth was clearly superior in the MES membrane. These in vivo essays proved that MES 


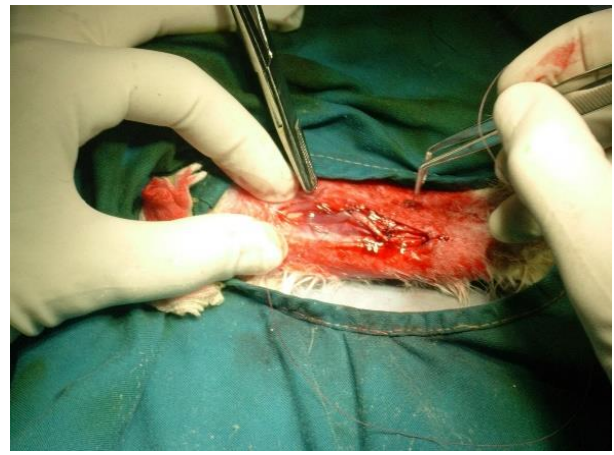

Figure 5. Experimental surgery on lab rabbit.

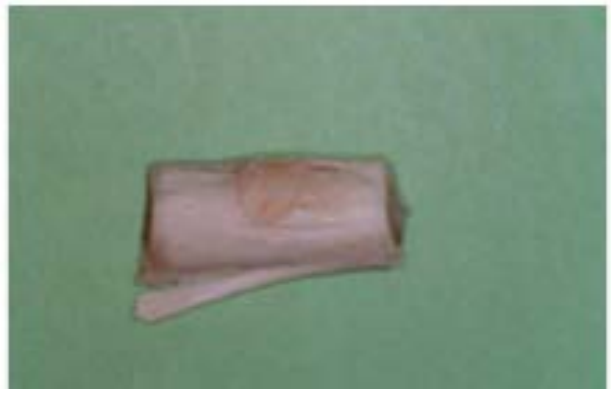

Figure 6. Histological sample of the rabbit after euthanasia.

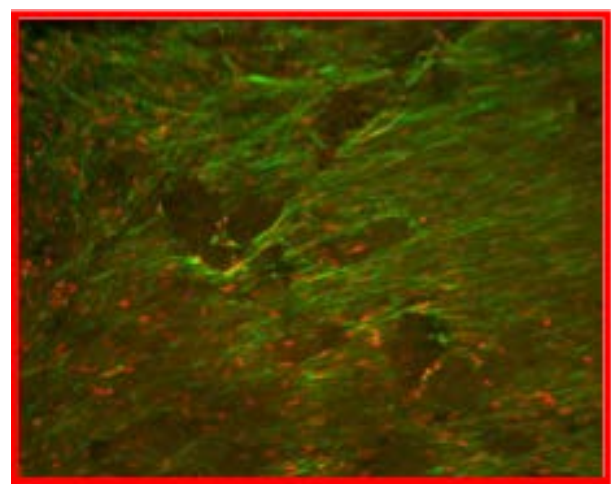

Figure 7. Confocal microscopy, MES membrane at 35 days. Cell nuclei (red) and cell cytoskeleton (green).

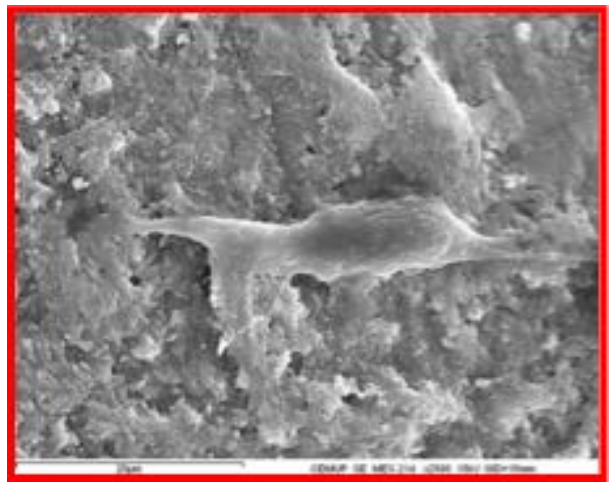

Figure 8. Colonization of osteoblasts on the surface of MES membrane after 21 days, MEV 2500×. 


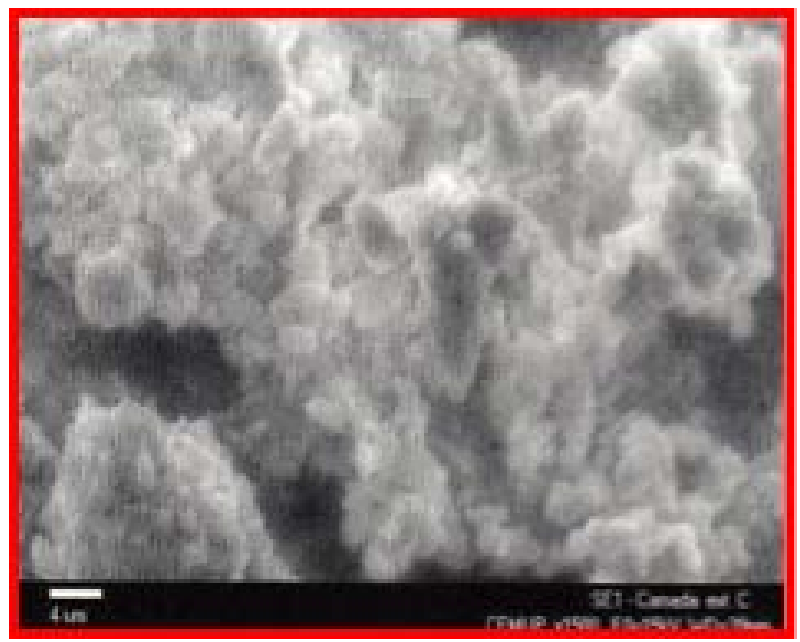

Figure 9. Mineralization on MES membrane after 7 days of immersion in synthetic plasma, MEV 1500×.

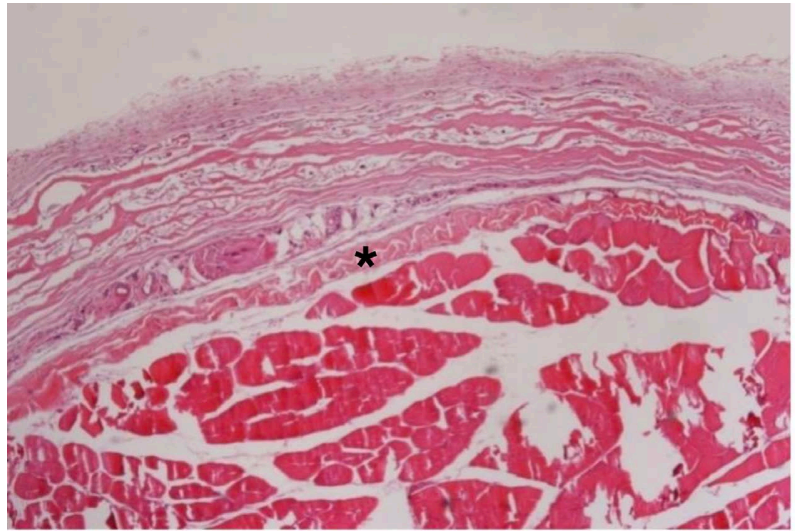

Figure 10. MES thin membrane $\left(^{*}\right)$ within the subcutaneous tissue, sample at 8 weeks, MO HE staining $40 \times$.

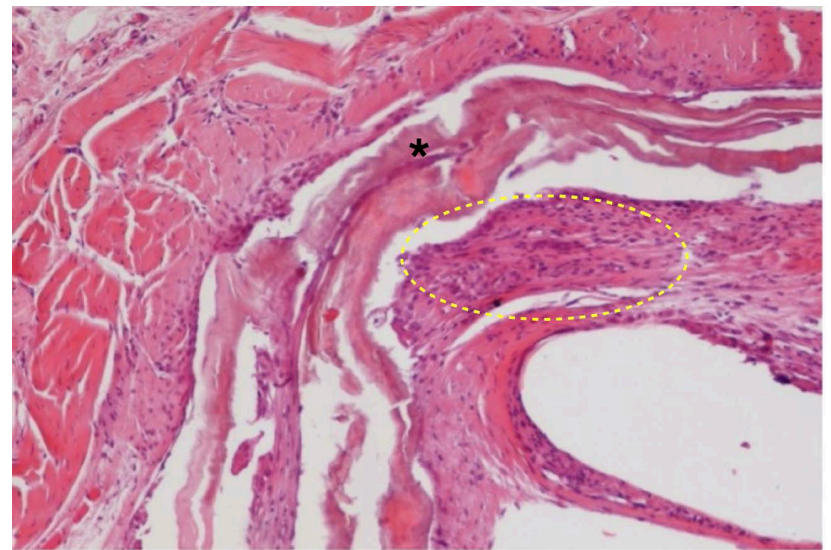

Figure 11. Thick MES membrane $\left(^{*}\right)$ with surrounding inflammatory infiltrate (yellow), MO HE staining $100 \times$.

membrane permit osteoblastic colonization and growth throughout its surface, proving to be highly histophilic and biocompatible. After the immersion in 
modified synthetic plasma for a week of the MES membrane, a mass mineralization throughout the membrane's surface occurred. This is coherent with the fact that the MES membrane is bioactive. The analyses of histological samples from experimental surgery in mice showed that the MES membrane wasn't toxic as far as soft tissues were concerned, that it provoked a moderate inflammatory response soon vanishing from the surrounding tissues after some time and it showed the first resorption signs after 8 weeks. In comparison with ACE membrane, this one had less inflammatory response than the MES membrane, since it is formed by autologous material (collagen) (Figure 12).

The analyses of histological (Figure 13, Figure 14) samples from experimental surgery in rabbits showed that the MES membrane induced guided bone regeneration in the created bone defect in a faster and more effective way than the physiological regeneration that occurred in the control bone defect (bone window). Furthermore, the membrane allows a bone growth in both surfaces (internal and external), which is compatible with osteoconductive and osteoinductive properties.

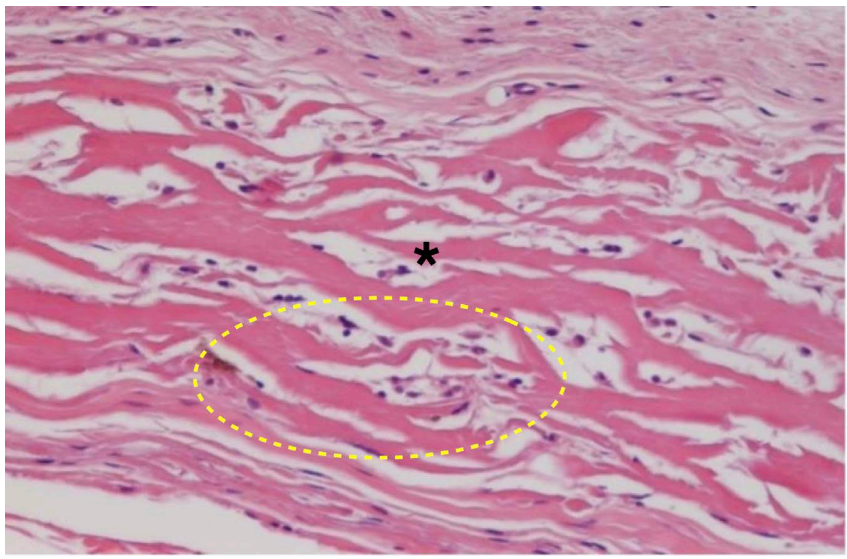

Figure 12. MES membrane ${ }^{*}$ ) with incipient signs of resorption (presence of inflammatory cells inside (yellow)), 8-week sample, MO HE staining 200x.

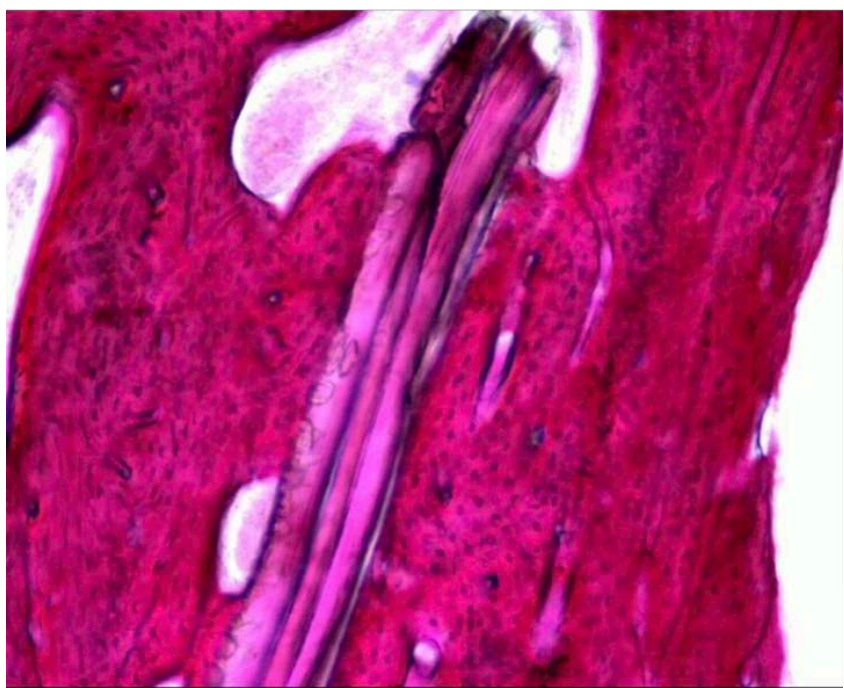

Figure 13. MES membrane on hard tissue of lab rabbit, MO 25×, HE staining. 


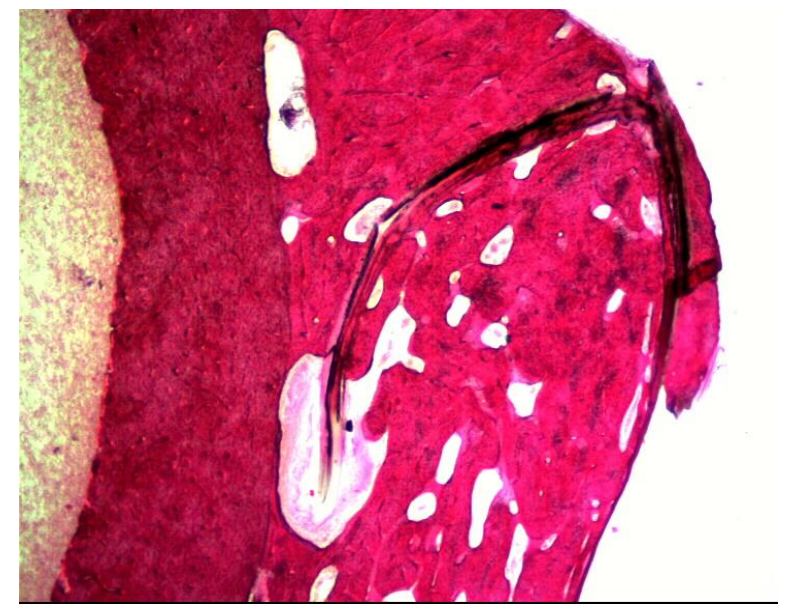

Figure 14. MES membrane on hard tissue of lab rabbit, MO 100×, HE staining.

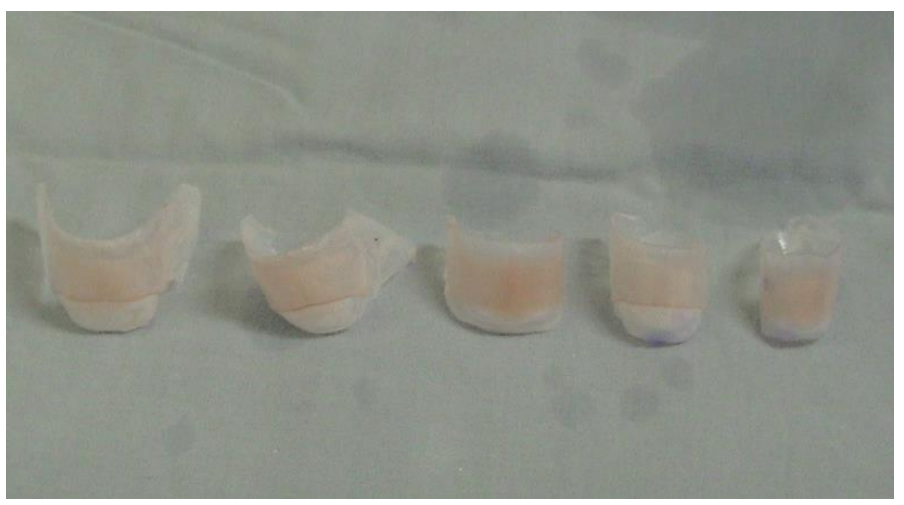

Figure 15. Several sizes of MES membrane.

This study also confirmed the first signs of membrane resorption after 8 weeks. After the immersion in modified synthetic plasma for a week of the MES membrane, a mass mineralization throughout the membrane's surface occurred. This is coherent with the fact that the MES membrane is bioactive.

\section{Conclusion}

The results obtained till now indicate that the MES biomaterial herein described proved to be biocompatible and non-toxic and can provide a promising functional therapeutic approach for Guided Bone Regeneration through its iterative "tent effect" design (Figure 15). Although the study is in an experimental pilot phase, the incorporation of MES membrane may further improve effective bone regeneration to satisfy all the requisites for clinical use as a GBR material.

\section{Conflicts of Interest}

There is no conflict of interest.

\section{References}

[1] Dimitriou, R., Mataliotakis, G.I., Calori, G.M. and Giannoudis, P.V. (2012) The 
Role of Barrier Membranes for Guided Bone Regeneration and Restoration of Large Bone Defects: Current Experimental and Clinical Evidence. BMC Medicine, 10, 81. https://doi.org/10.1186/1741-7015-10-81

[2] Nevins, M. and Mellonig, J.T. (2003) Implantoterapia-Abordagens Clínicas e Evidência de Sucesso. Quintessence editora ltda, São Paulo, 53-72.

[3] Lang, N.P., Araújo, M. and Karring, T. (1997) Alveolar Bone Formation. In: Llndhe, J., Karring, T. and Lang, N.P., Eds., Clinical Periodontology and Implant Dentistry, International Publishers Ltd., Copenhagen.

[4] Boyne, P.J. (1997) Osseous Reconstruction of the Maxilla and the Mandible: Surgical Techniques Using Titanium Mesh and Bone Mineral. Quintessence Publishing Co., Chicago. https://doi.org/10.1097/00008505-199807040-00022

[5] Lundgren, D., Nyman, S., Mathisen, T., Isaksson, S. and Klinge, B. (1992) Guided Bone Regeneration of Cranial Defects, Using Biodegradable Barriers: An Experimental Pilot Study in the Rabbit. Journal of Cranio-Maxillofacial Surgery, 20, 257-260. https://doi.org/10.1016/S1010-5182(05)80438-X

[6] Wang, H.L. and Macneil, R.L. (1998) Guided Tissue Regeneration. Absorbable Barriers. Dental Clinics of North America, 42, 505-522.

[7] Patino, M.J., Neiders, M.E., Andreana, S., Noble, B. and Cohen, R.E. (2002) Collagen as an Implantable Material in Medicine and Dentistry. Journal of Oral Implantology, 28, 220-225. https://doi.org/10.1563/1548-1336(2002)028<0220:CAAIMI >2.3.CO;2

[8] Zitzmann, N.U., Naef, R. and Scharer, P. (1997) Resorbable versus Nonresorbable Membranes in Combination with Bio-Oss for Guided Bone Regeneration. International Journal of Oral \& Maxillofacial Implants, 12, 844-852.

[9] Elharar, F., Rodriguez, H.J., Benque, E.P. and Caffesse, R.G. (1998) Guided Tissue Regeneration with Bioabsorbable and Expanded Polytetrafluoroethylene Barrier Membranes in the Treatment of Naturally Occurring Periodontal Defects in Dogs. Journal of Periodontology, 69, 1218-1228. https://doi.org/10.1902/jop.1998.69.11.1218

[10] Aaboe, M., Pinholt, E.M., Schou, S. and Hjorting-Hansen, E. (1998) Incomplete Bone Regeneration of Rabbit Calvarial Defects Using Different Membranes. Clinical Oral Implants Research, 9, 313-320. https://doi.org/10.1034/j.1600-0501.1998.090504.x

[11] McGinnis, M., Larsen, P., Miloro, M. and Beck, F.M. (1998) Comparison of Resorbable and Nonresorbable Guided Bone Regeneration Materials: A Preliminary Study. International Journal of Oral \& Maxillofacial Implants, 13, 30-35. https://doi.org/10.1016/S0266-4356(97)90742-7

[12] Bunyaratavej, P. and Wang, H.L. (2001) Collagen Membranes: A Review. Journal of Periodontology, 72, 215-229. https://doi.org/10.1902/jop.2001.72.2.215

[13] Mesquita, P., Branco, R., Afonso, A., Vasconcelos, M. and Cavalheiro, J. (2004) Mineralised Membranes for Bone Regeneration (Key Engineering Materials). Biodental Engineering IV, 254-256, 1091-1094.

https://doi.org/10.4028/www.scientific.net/KEM.254-256.1091

[14] Simões, R., Fonseca, L., Vasconcelos, M., Afonso, A. and Cavalheiro, J. (2002) Modified Exoskeleton of Shrimp for Guided Bone Regeneration. The 17 th ESB, Barcelona, September 2012, 35. 